\title{
Low error estimation of half-cell thermodynamic parameters from whole-cell Li-ion battery experiments: Physics-based model formulation, experimental demonstration, and an open software tool
}

\author{
Victor.W. Hu, and Daniel. T. Schwartz \\ Department of Chemical Engineering \\ Clean Energy Institute \\ University of Washington \\ Seattle, Washington 98195-1750, USA \\ dts@uw.edu
}

\begin{abstract}
Low C-rate charge and discharge experiments, plus complementary differential voltage or differential capacity analysis, are among the most common battery characterization methods. Here, we adapt the multispecies, multi-reaction (MSMR) half-cell thermodynamic model to low C-rate cycling of whole-cell Li-ion batteries. MSMR models for the anode and cathode are coupled through whole-cell charge balances and cell-cycling voltage constraint equations, forming the basis for model-based estimation of MSMR half-cell parameters from whole-cell experimental data. Emergent properties of the whole-cell, like slippage of the anode and cathode lithiation windows, are also computed as cells cycle and degrade. A sequential leastsquare optimization scheme is used for parameter estimation from low-C cycling data of Samsung 18650 $\mathrm{NMC} \mid \mathrm{C}$ cells. Low-error fits of the open-circuit cell voltage (e.g., under $5 \mathrm{mV}$ mean absolute error for charge or discharge curves) and differential voltage curves for fresh and aged cells are achieved. We explore the features (and limitations) of using literature reference values for the MSMR half-cell thermodynamic parameters (reducing our whole-cell formulation to a 1-degree-of-freedom fit) and demonstrate the benefits of expanding the degrees of freedom by letting the MSMR parameters be tailored to the cell under test, within a constrained neighborhood of the half-cell reference values. Bootstrap analysis is performed on each dataset to show the robustness of our fitting to experimental noise and data sampling over the course of 600 cell cycles. The results show which specific MSMR insertion reactions are most responsible for capacity loss in each half-cell and the collective interactions that lead to whole-cell slippage and changes in useable capacity. Open-source software is made available to easily extend this model-based analysis to other labs and battery chemistries.
\end{abstract}




\section{Introduction}

Widespread electrification of new economic sectors is driving the demand for reliable, safe, and affordable high energy and power-density batteries. To meet this demand, battery materials are continually advancing, separators are getting thinner, the ratio of electrolyte to active material is falling, and ever larger cell formats are being introduced. ${ }^{1}$ The reality of these modern battery engineering strategies is that the response of an engineered cell increasingly requires the analysis of two strongly-interacting active electrodes, not simplified half-cells. Nonetheless, battery science is largely half-cell science carried out in small button cell (CR2032) geometries, providing key properties of pristine active materials without the complicating, but important, implications of a second active electrode microns away. ${ }^{2-4}$

Deconvoluting fundamental physicochemical parameters for each electrode during operation of an engineered whole-cell is critical for understanding device performance, especially aging, but it is fundamentally challenging ..$^{5-8}$ The differences between characterizing battery half-cells and whole-cells can be profound: coin cells use excess electrolyte whereas engineered whole-cells are lean on electrolyte; the two classes of cells have different thermal and current distribution behaviors, and it also matters if the whole cell is a cylindrical, pouch, or prismatic configurations under (nominally) identical electrochemical testing. ${ }^{2,9}$ One way to extract half-cell electrochemical data from engineered whole-cells is destructive analysis. In this case, the (usually aged) whole-cell is disassembled to harvest electrodes for more traditional half-cell studies and materials analysis, but this introduces uncertainties such as cell compression effects, differences in electrolyte composition and amount, and possible damage to electrode surfaces and pores structure. $^{10}$ The alternative is to apply appropriate physics-based modeling to analyze whole-cell measurements, then use sophisticated estimation methods to determine fundamental physicochemical parameters of the half cell.

Here we adapt a half-cell physics-based thermodynamic model, and share open-source software, that can be used to parameterize whole-cell measurements, resulting in low residual error half-cell data. For insertion electrodes, solid-state thermodynamics dictate material phases and reactions that underpin the potential achievable at any given state-of-charge. ${ }^{11}$ The thermodynamic open-circuit voltage (OCV) in a battery is the electrochemical potential difference between the anode and the cathode in a common electrolyte. ${ }^{8}$ Common experimental techniques to measure the OCV at different intercalation states involve low C-rate galvanostatic cycling ${ }^{12-14}$, complementary differential voltage (dV/dQ) or differential capacity $(\mathrm{dQ} / \mathrm{dV})$, as well as galvanostatic or potentiostatic intermittent titration techniques. ${ }^{15,16}$ Because an engineered whole-cell operates without a reference electrode, OCV vs. state-of-charge data alone is insufficient to estimate potentials of either electrodes against a known thermodynamic reference.$^{7,17,18}$ However, we show that whole-cell OCV measurements combined with a physics-based solid-state 
thermodynamic model that is "seeded" with half-cell parameters referenced to lithium metal, can be used to self-consistently decouple and quantify individual electrode thermodynamic parameters from the wholecell response. We offer a quantitative approach that goes beyond using half-cell studies as a basis for assigning peaks in whole-cell differential analysis ${ }^{19-24}$, while avoiding destructive postmortem analysis. ${ }^{25}$ Whole cell parameter estimation using differential voltage and differential capacity data from aging cells

helps understand inhomogeneous material degradation and slippage. ${ }^{26-28}$ Open software used for the analysis of engineered whole-cells is provided as a Jupyter notebook.

\section{Physics-Based Modeling and Parameter Estimation Approach}

Thermodynamic model attributes - The Multi-Species, Multi-Reactions (MSMR) model describes the electrochemical thermodynamics of solid-state reactions and phase transitions that insertion materials go through at different lithiation states. ${ }^{29-31}$ The model has been shown to nicely match experimental halfcell open-circuit potential data, and it captures a wide range of solid-state complexity, including phase changes. Because the model has a simple deterministic form with easily interpretable parameters, it can be tuned to explore the effects of parameters on OCP and differential voltage and, as shown here, used in optimization software for robust parameter estimation. Studies of open-circuit and differential voltage spectra of whole-cells typically build from half-cell experimental data and models. ${ }^{32-34}$ As a result of having physically interpretable parameters, the MSMR model has been used to gain insight into interfacial resistances in graphite ${ }^{35,36}$, interfacial reactions in $\mathrm{Li}_{-} \mathrm{Si}^{31}$, and the effect of scan rate in linear sweep voltammetry experiments ${ }^{29,30}$.

Mathematically, the MSMR model describes potential-dependent lithium occupancy of different insertion reactions in a half-cell electrode under study (each reaction is denoted by subscript $j$ ). To directly align with experimental quantities, we write the MSMR model with extensive variables, representing the inserted $\mathrm{Li}^{+}$charge with Q (Ah units), rather than site occupancy fraction (an intensive variable):

$$
Q_{j}(U)=\frac{Q_{j, t o t}}{1+\exp \left[f\left(U-U_{j}^{0}\right) / \omega_{j}\right]}
$$

and differential capacity

$$
\frac{d Q_{j}}{d U}(U)=-\frac{Q_{j, t o t}}{\omega_{j}} \frac{f \exp \left[f\left(U-U_{j}^{0}\right) / \omega_{j}\right]}{\left[1+\exp \left[f\left(U-U_{j}^{0}\right) / \omega_{j}\right]\right]^{2}}
$$

where $f=F /(R T), U$ is the half-cell potential (vs. a lithium metal reference), $U_{j}^{0}$ is the standard potential of any reaction $j$, and $\omega_{\mathrm{j}}$ captures non-ideality associated with intercalation reaction $j$. Ideal Nernstian behavior is represented by $\omega_{j}=1$, whereas attractive interactions in the lithiated solid occurs with $\omega_{j}<1$. Strong 
attractive interactions can represent complex phase behavior such as staging seen in graphite anodes. Alternatively, repulsive interaction occurs with $\omega_{j}>1$. Each of the $j$ reactions has a total insertion capacity denoted $Q_{j, t o t}$. To calculate the overall insertion capacity and differential capacity of an electrode at any given voltage, the set of independent intercalation reactions are summed over all $\mathrm{J}$ reactions that occur in the insertion half-cell:

$$
Q(U)=\Sigma_{1}^{J} Q_{j}(U)
$$

and

$$
\frac{d Q}{d U}(U)=\sum_{1}^{J} \frac{d Q_{j}}{d U}(U)
$$

The total insertion capacity of all electrode reactions is

$$
Q_{t o t}=\sum_{1}^{J} Q_{j, t o t}
$$

The MSMR half-cell equations work equally well for the typical positive and negative insertion electrodes used in many Li-ion chemistries, and later we add a (+) or (-) superscript to variables and parameters to assign them to a particular electrode in the whole-cell.

Utilizing half-cell models to create a whole-cell response - Qualitatively, the difference between half-cell and whole-cell models is the coupling of charge transfer in each electrode, where incremental insertion in one electrode $(\delta Q)$ is complemented, ideally, by an equal and opposite extraction from the other electrode. For safety and durability, this simultaneous insertion/extraction process is bounded by upper and lower whole-cell voltage limits. Consequently, for typical Li-ion chemistries, positive electrodes are cycled between intermediate lithiation states ${ }^{37}$, whereas graphite anodes cycle to near their fully-delithiated state. The usable capacity $(\Delta Q)$ achieved by cycling between the upper (charged) and lower (discharged) cell voltage limits is lower than the total insertion capacity of either the positive or negative electrode (denoted $Q_{t o t}^{+}$and $Q_{t o t}^{-}$, respectively) as defined in Eq. (5).

Mathematically, the relationship between each half-cell's lithiation state at the upper voltage limits can be written

$$
V_{u p p e r}=U^{+}\left(Q_{\min }^{+}\right)-U^{-}\left(Q_{\max }^{-}\right)
$$

for a nominally charged cell, and for the lower cell voltage

$$
V_{\text {lower }}=U^{+}\left(Q_{\max }^{+}\right)-U^{-}\left(Q_{\min }^{-}\right)
$$


in a nominally discharged cell. The superscripts $(+)$ and (-) denote the positive and negative half-cell variables computed from Eqs. (1)-(5), and the subscripts $\max$ and $\min$ describe the maximum and minimum inserted lithium ion in each electrode during a single charge/discharge cycle. The useable charge capacity $(\Delta Q)$ of an ideal cell operating between the specified upper and lower voltage bounds is defined by the relationship

$$
Q_{\max }^{ \pm}=Q_{\min }^{ \pm}+\Delta Q
$$

for both the positive and negative electrodes. Of course, batteries are not ideal and age over many cycles (by many mechanisms), slowly reducing the useable charge, resulting in a shift of $Q_{\text {max }}^{ \pm}$and $Q_{\min }^{ \pm}$to simultaneously satisfy the constraints set by Eqs. (6)-(8) with $V_{\text {upper }}$ and $V_{\text {lower }}$ fixed. The consequences of loss of capacity, and the resulting "slippage" of the lithiated states for nominally fully-charged and fullydischarged cells propagate through the estimation of all parameters in Eqs. (1)-(8).

Formulating a charge conserving full-cell model to optimize against experimental data Experimentally, we use standard cell testing methods, namely, low-rate constant-current charge and discharge (low C-rate), to determine the equilibrium cell voltage over the accessible range of lithiation states between the discharged and charged cell. Experimental whole-cell voltages, at any state-of-charge, are represented in model variables as

$$
V=U^{+}\left(Q^{+}\right)-U^{-}\left(Q^{-}\right)
$$

where the measured voltages are related to state-of-charge in each electrode. Each increment of measured charge $\left(\delta Q^{\exp }\right)$ has a concomitant Li-ion insertion/extraction charge, such that $\delta Q^{\exp }=\delta Q^{+}=-\delta Q^{-}$for ideal faradaic efficiency. The differential voltage is also often used as a highly sensitive method for identifying multiple solid-state insertion reactions. With the nomenclature of this work, differential voltage experiments can be represented as:

$$
\frac{d V}{d Q}=\frac{d U^{+}\left(Q^{+}\right)}{d Q}+\frac{d U^{-}\left(Q^{-}\right)}{d Q}
$$

with the continuing assumption of ideal faradaic efficiency for a given cycle.

The MSMR model, Eq. (1)-(5), uses half-cell potential as the independent variable, with extent of solid lithiation as the dependent variable, whereas typical experiments, represented by Eq. (9) and (10), control lithiation state and measure the resulting voltage. Though the natural independent and dependent variables from modeling and experiments are not identical, both formulations behave well when inverted (since voltage and charge are monotonic functions in Li-ion batteries). Thus, there are several options for 
formulating the optimization problem when seeking to estimate model parameters against data. As represented in the Jupyter Notebook used for this work ${ }^{38}$, we have found robust model fitting to experimental data from minimization of the Cost function,

$$
\text { Cost }=\text { weight }_{1} \sum_{k} \frac{\left|\delta Q_{k}^{\text {exp }}-\delta Q_{k}^{M S M R}\right|}{\left\langle\delta Q^{\exp }\right\rangle}+\text { weight }_{2} \sum \frac{\left|\left(\frac{d V}{d Q}\right)_{k}^{\text {exp }}-\left(\frac{d V}{d Q}\right)_{k}^{M S M R}\right|}{\left\langle\frac{d V^{\exp }}{d Q}\right\rangle}
$$

as a function of cell voltage, where the brackets $<>$ denote average values. In the initial work, we used 1000 evenly spaced points in voltage to define incremental charge and differential voltage in the experiment and modeled system (even spacing was relaxed in later bootstrap analysis). Moreover, if we assume the experimentally measured useable charge $\Delta Q^{\exp }$ is ideally related to the modeled insertion/extraction in any given cycle, so that,

$$
\Delta Q^{\exp }=\Delta Q^{+}=-\Delta Q^{-}
$$

then we can use $Q_{\min }^{+}$and $Q_{\min }^{-}$as parameters of the model and apply Eq. (8) to determine $Q_{\max }^{ \pm}$for the positive and negative electrodes. We also constrain our fits by enforcing Eq. (6) and (7), so that model and experiments agree exactly at the upper and lower cell voltages

$$
V_{\text {upper }}^{\text {exp }}=U^{+}\left(Q_{\text {min }}^{+}\right)-U^{-}\left(Q_{\max }^{-}\right)
$$

and

$$
V_{\text {lower }}^{\text {exp }}=U^{+}\left(Q_{\max }^{+}\right)-U^{-}\left(Q_{\min }^{-}\right)
$$

Finally, if the total positive and negative electrode capacities are known reliably by independent experimental measurements, i.e., $Q_{\text {tot }}^{\text {exp }}$ is known for both electrodes, then Eq. (5) can be used to constrain the modeled values of $Q_{j, t o t}$ for each electrode by enforcing an exact match to the experimental values. When doing parameter estimation, we allowed total capacity of both electrodes to be determined by the fit, rather than imposed as a constraint. This choice has several reasons and consequences. First, we are using commercial cells for our experiments, with imprecisely known total active material loading, so it is unclear how accurately we can know $Q_{\text {tot }}^{\exp }$. Second, as cells degrade with cycling, the total capacity of each electrode evolves into an unknown, even if there is good data on the fresh cell. Finally, determining the total capacity based on the fit parameters can allow a physics-informed evaluation of the reasonableness of the fitting process.

Seeding initial parameters and establishing physical bounds for parameters. - The procedures for initiating the best-fit of half-cell parameters $\left(Q_{j . t o t}, U_{j}^{0}, \omega_{j}\right)$ in fresh cells begins with reference parameters 
determined by Verbrugge et al. ${ }^{39}$ for a variety of cathode and anode materials measured in half-cells. As shown in the Results and Discussion section, the reference parameters give reasonable representations of the carbon and blended NMC/LMO electrodes in our experimental cell, when normalized by an appropriate initial guess for the positive and negative electrode total capacities $Q_{t o t}$, along with enforcement of conditions (8), (12)-(14) at the beginning and end of discharge. Because the MSMR model parameters are physically intuitive and have easily predicted effects on half-cell behavior, we normally make small user adjustments to the reference parameter set to help ensure rapid convergence to low mean absolute error fits of the fresh cell experimental data. Fitting bounds were placed on each type of parameter: $U_{j}^{0}$ was allowed to vary by $20 \mathrm{mV}$ from the initial value; $Q_{j, t o t}$ was allowed to vary by $25 \%$ of the initial value, and $\omega_{\mathrm{j}}$ was allowed to vary by $25 \%$ of the initial value. The one exception was allowing only $5 \%$ variation on the $Q_{j, t o t}$ parameters for the LMO reactions, to ensure the proportion of NMC to LMO in the blended electrode was close to the value we measured experimentally.

Our implementation of parameter estimation (see Jupyter notebook ${ }^{38}$ ) involves Cost function minimization using fmin_slsqp, a sequential least-squares optimization scheme in the Python package SciPy. We evenly weight the Cost function between the capacity and differential voltage terms in Eq. (11). Our basic procedure is to optimize over 1000 evenly spaced points in voltage over the range of $3.49 \mathrm{~V}$ to $4.15 \mathrm{~V}$. This optimization window is less than the lower and upper cell voltage cycling limits (set as $V_{\text {lower }}$ $=2.56 \mathrm{~V}$ and $V_{\text {upper }}=4.2 \mathrm{~V}$ in the charging experiments described below). By limiting the Cost function minimization to this window, the fit focuses on the major features in the differential voltage data, without overly biasing the fit by extreme differential voltages seen at the boundaries of the charging curves. Nonetheless, the full cycling window is part of the optimization through the constraints set by Eqs. (8), (12) - (14), ensuring that the modeled full-cell voltages and usable charge matches the experimental voltages at the beginning $(2.56 \mathrm{~V})$ and end $(4.2 \mathrm{~V})$ of a charge cycle.

When doing a full parameter estimation, with model parameters $U_{j}^{0}, Q_{j, t o t}$ and $\omega_{j}$ determined for low C-rate charging of fresh cells, those parameters become the initial guesses for fitting the next dataset after the cells are aged. Here we reevaluated the cells after 300 aggressive cycles. For data from 300 aging cycles (and all subsequent cycles), the tight bounds on LMO capacity parameters are relaxed to match the $\pm 25 \%$ bounds on NMC values of $Q_{j, t o t}$. Likewise, the best-fit model parameters from 300 cycles become the initial guess for fitting low C-rate testing after 600 total cycles. For the fits of the 600-cycle aged cell, the $U_{j}^{0}$ parameters are bounded within $\pm 10 \mathrm{mV}$ of the values 300 cycle best-fit values.

Because the experimentally measured useable capacity declines during cell cycling, Eqs. (8), (12)(14) show the fundamental origin of so called "slippage" in Li-ion batteries. Slippage arises naturally when there is model agreement with the experimental voltage constraints and (changing) useable charge values. 
Here, we allow the minimum inserted lithium in each electrode, $Q_{\min }^{+}$and $Q_{\min }^{-}$, to provide the degrees of freedom that allow the meeting of constraints at the upper and lower voltage bounds of our experimental measurements. For the positive electrode, we set an upper bound for fitting of $Q_{\min }^{+}$to the value from the last best-fit of data. The lower bound for $Q_{\min }^{+}$is set as if all the measured experimental capacity loss is in slippage of $Q_{\min }^{+}$; the best fit must fall between these physically-reasonable bounds. For the negative electrode, the fitting bounds were set between fully delithiated carbon and $0.5 \%$ lithiated carbon (for our fresh cells, that means $0 \leq Q_{\min }^{-} \leq 0.0108 \mathrm{Ahr}$ ).

Bootstrapping Analysis.-To further explore the statistical sensitivity of our analysis to details of experimental sampling and noise, we performed a bootstrapping analysis. Instead of using 1000 evenly spaced points within the optimization voltage range, the bootstrapping process takes 1000 random points (out of roughly 6000 for each dataset), and then performs the parameter estimation, repeating the process (with replacement) through 500 iterations. These bootstrapped data are first filtered to remove any fit iterations with differential voltage MAE $>0.04 \mathrm{~V} / \mathrm{Ahr}$, as these represented outlier fits that do not accurately capture the features of the datasets. These filtered data are then used to calculate the population median and confidence interval for each parameter. All data shown in the main body of this manuscript are computed and fit against experimental charging data from the lower voltage limit to the upper voltage limit. In the Supplementary Information, the complementary experimental data for cell discharge, discharge best-fit parameters, and bootstrapped results are presented.

\section{Experimental Methods}

Cycling experiments were performed on commercially available Samsung 1.5 Ahr LiNMC |C cells (INR 18650-15M) using a Maccor 4000M battery cycler in a Maccor MTC-020 environment chamber set at $25^{\circ} \mathrm{C}$. All cells were first subjected to five initial cycles with a $\mathrm{C} / 2$ charge and discharge rate within the specified voltage windows ( $2.5 \mathrm{~V}$ to 4.2$)$, where the cell capacity stopped increasing. To evaluate cell degradation in these batteries, individual cells were cycled to 300 and 600 cycles, where a full cycle included a $2.67 \mathrm{C}$ ( $4 \mathrm{~A}$ ) charge to $4.2 \mathrm{~V}$, a constant voltage hold at $4.2 \mathrm{~V}$ until current drops to $100 \mathrm{~mA}$, and then a $2.67 \mathrm{C}$ discharge down to $2.5 \mathrm{~V}$.

Once the batteries had completed the specified cycling profile, the cells were then discharged at a $\mathrm{C} / 10$ rate to $2.5 \mathrm{~V}$. The cells were then subjected to a low galvanostatically controlled $(\mathrm{C} / 20)$ charge and discharge to estimate the open-circuit voltage data, with data points collected every 10 seconds. To get the differential voltage (dV/dQ), a smoothing filter was applied for each dataset to reduce differentiation noise and error. ${ }^{40}$ A Savitzy-Golay filter with a third-order polynomial was applied around a central voltage with $\mathrm{n}$ data points in the window length, resulting in $(\mathrm{n}-1) / 2$ points before and after the central voltage.. The 
smoothed values at the edges were calculated from the polynomials fit at the first and last central voltages that satisfy the window length. After preliminary evaluation, we selected a value of $n=99$ points, resulting in a window with a central voltage that has 49 points before and after so that all the major features of the differential voltage were easily distinguishable, but not overly smoothed.

To estimate the total insertion capacities of the electrodes for the cells under test, we discharged the cells to $0.00 \mathrm{~V}$ and then opened the cell casing in a glove box to extract the electrodes and separator. The positive and negative electrode sheets were peeled from the separator and placed into a vacuum oven set at $80^{\circ} \mathrm{C}$ overnight to remove solvents. Cathode disks were punched from the dried sheet using a $15 \mathrm{~mm}$ arc punch and weighed. Active material from the cathode was removed using N-methyl-2-pyrrolidone (NMP, Sigma-Aldrich) to reveal the bare current collector, which was then dried overnight in the vacuum oven and weighed. The mass difference between the coated and bare current collector was the weight of the active material, binder, and other additives; standard ratios were used to estimate a total cathode insertion capacity of $Q_{t o t}^{+}=1.8 \mathrm{Ah}$. Anode materials were less reliably measurable, owing to poor adhesion of the coating to the current collector, resulting in material losses when peeled from the separator and when punched. Initial differential voltage analysis measurements indicated that these commercial NMC cells were a blended cathode system, composed of both $\mathrm{N}_{\mathrm{x}} \mathrm{M}_{\mathrm{y}} \mathrm{C}_{\mathrm{z}}$ and spinel $\mathrm{MnO}_{2}{ }^{25,41}$ This was confirmed with X-Ray diffraction (XRD) and energy dispersive X-ray spectroscopy (EDX) along with the composition of the $\mathrm{NMC}$ and fractional $\mathrm{MnO}_{2}$. Electrode capacities were then estimated from the mass and composition data combined with literature values for published capacities for $\mathrm{NMC}^{42-45}$, and $\mathrm{MnO}_{2},{ }^{42,46}$ along with common formulations for the ratio of active electrode material to binder and additives .

\section{Results and Discussion}

Verbrugge et al., determined MSMR parameters for a wide variety of insertion electrodes based on fits to half-cell experiments. For our cells under test, X-ray diffraction and energy dispersive X-ray analysis showed that the cathode material is a blend of $66 \%$ NMC532 and $34 \%$ spinel- $\mathrm{MnO}_{2}$, combined with a graphite anode. Verbrugge et al. have provided best-fit half-cell parameters for NMC 622, LMO, and carbon, and thus, provides a good initial set of thermophysical parameters for our cathode and anode material classes. The MSMR model easily accommodates a blended electrode, since the total capacity is additive at a given potential, per Eqs. (1) and (5). Consequently, the appropriate reactions for both LMO and NMC were added together, using reaction capacities commensurate with the measured blending ratio, to achieve the half-cell thermodynamics of the cathode.

Implementing the MSMR half-cell model into the whole-cell paradigm.- To illustrate how experimental whole-cell usable charge and voltage constraints, Eqs. (8), (12)-(14) are implement with 
MSMR half-cell models, we start by describing a single degree of freedom fitting of the model-tomeasurements, then successively relax assumptions to achieve a higher degree of freedom best-fit of modelto-measurements.

To reduce the model degrees of freedom, we use the MSMR half-cell thermophysical parameters reported in Verbrugge et al. to represent our blended NMC/LMO (66/34) cathode and graphite anode; this removes all the MSMR model parameters as unknowns (there are six insertion reactions per electrode). Further, we scale the thermophysical parameters with an estimate of total insertion capacity for each electrode, $Q_{t o t}^{ \pm}$in the experimental cell under test. Specifically, we estimate the total cathode capacity $Q_{\text {tot }}^{+}=1.8 \mathrm{Ah}$ for our $1.5 \mathrm{Ah}$-rated Samsung cells, based on weighing of the cathode loading in disassembled cells, along with reasonable estimates of binder and other additive masses, as well as specific capacities for the blended cathode materials $(200 \mathrm{mAh} / \mathrm{g}$ and $120 \mathrm{mAh} / \mathrm{g}$ for NMC532 and LMO, respectively), A typical $\mathrm{n} / \mathrm{p}$ ratio of 1.1 was used to estimate the negative electrode capacity $Q_{\text {tot }}^{-}=$ $1.96 \mathrm{Ah}$.

With these half-cell and cell-under-test parameters estimated through independent means, and not allowed to vary, the whole-cell MSMR model has a single degree of freedom — chosen from among the parameters, $\mathrm{Q}_{\min }^{+}, \mathrm{Q}_{\max }^{+}, \mathrm{Q}_{\min }^{-}, \mathrm{Q}_{\max }^{-}$- to fit the measured usable charge $(\Delta Q=1.48 \mathrm{Ah})$ from $\mathrm{C} / 20$ experiment charging from a cell voltage of $2.56 \mathrm{~V}$ to $4.2 \mathrm{~V}$. We chose to vary $\mathrm{Q}_{\min }^{+}$and used Eqs. (8), (12)-(14), along with the MSMR model (and Verbrugge et al. parameters), to search for a model that matched with whole cell experimental data. It was not known a priori whether the highly constraining set of modeling assumptions and estimated values used to reduce the whole-cell MSMR model to a single degree-of-freedom would produce one, none, or multiple fits to the whole-cell data. Perhaps surprisingly, we found a single value of $\mathrm{Q}_{\min }^{+}$for the model that permitted all whole-cell equations (8), (12)-(14) to be satisfied between the experimental voltage window and measured usable charge. The single experimentally-aligned whole-cell fit of the MSMR model parameters is shown in Figure 1 as the differential capacity $(\mathrm{dQ} / \mathrm{dU})$ for each electrode as a function of the half-cell potential $\mathrm{U}$ vs. $\mathrm{Li}^{+} / \mathrm{Li}$ reference.

Figure 1 is a representation of the MSMR model that shows the density of lithiation states in each electrode as a function of potential (against the reference electrode). Filled lithiation states in the blended cathode (red, at more positive potentials) and graphite electrodes (blue, at more negative potentials) are shown as shaded areas in the discharged state with cell voltage of $2.56 \mathrm{~V}$, Figure 1(a), and the charged state, Figure 1(b), with cell voltage of $4.2 \mathrm{~V}$. The quantity of charge extracted from the cathode in going from the lower-to-upper cell voltage matches the experimental usable capacity (1.48 Ah) measured in the fresh 
Samsung cells. Likewise, the charge inserted into the anode going from the lower-to-upper voltage is equal and opposite to the extracted charge and matches the experiments for a fresh cell. The cell voltage in the discharged and charged states has half-cell potentials at each lithiated state of the electrodes that, when subtracted, exactly match the lower and upper cell voltage bounds used in the experiments. Thus, Eqs. (8), (12)-(14) can be satisfied when we use literature parameters for the MSMR model and our experimental estimates of each electrode's total capacity. Based on the Verbrugge et al. model parameters and wholecell values described here, we found the minimum lithiation for the positive electrode to be $0.185 \mathrm{Ahr}$ $\left(\mathrm{Q}_{\min }^{+}\right)$and $0.001 \mathrm{Ahr}$ for the negative electrode $\left(\mathrm{Q}_{\min }^{-}\right)$, with the maximum values simply being $1.48 \mathrm{Ah}$ greater, per Eq. (8).

For the blended cathode system, Fig. 1 shows that the peaks in the differential capacity are broad, indicative of single-phase reactions. It has been shown that NMC follows this behavior, but the reactions at

(a)

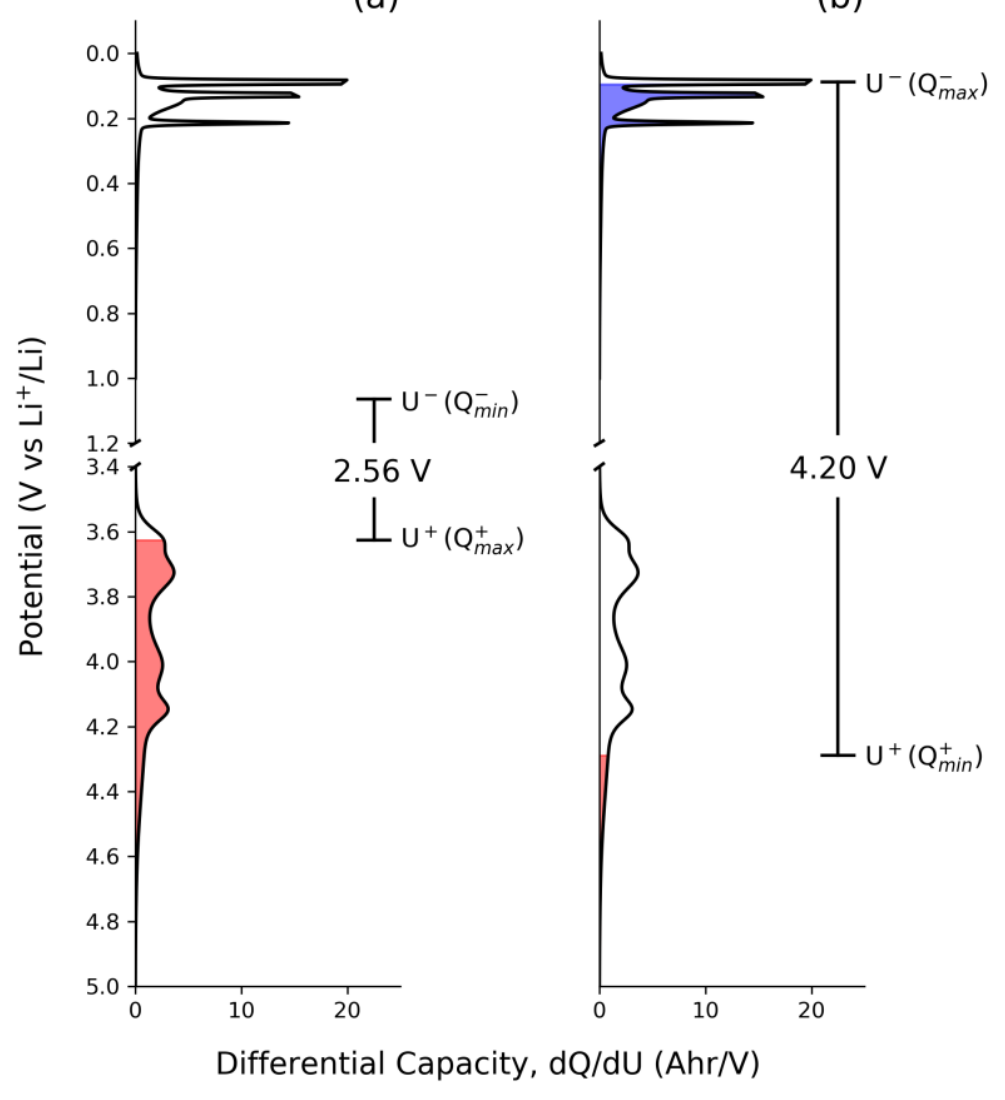

Figure 1 Differential capacity of a simulated blended Li NMC $\mid \mathrm{MnO}_{2}$ cathode (bottom) and graphite cathode (top ) in a discharged (a) and charged (b) state that meets the voltage limit constraints in our experimental data. The shaded portions denote the lithiated capacity in the positive electrode (red) and the negative electrode (blue) at each state of charge. The maximum differential capacity for the anode is more than an order of magnitude larger than the cathode maximum, so anode peaks are cut-off for clarity; this makes it difficult to see that the quantity extracted charge from the cathode exactly equals the inserted charge for the anode in going from (a) to (b). 
4.0 and $4.15 \mathrm{~V}$ are typically two-phase reactions caused by the redistribution of the lithium ions in the LMO material. ${ }^{8,22,47}$ However purely crystalline, two-phase behavior is hard to maintain for real world applications of LMO, leading the electrode reactions to exhibit more single-phase behavior. ${ }^{39}$ In contrast, the differential capacity of the graphite electrode has very sharp peaks, due to the different staging reactions that store a large fraction of capacity over a narrow potential range. ${ }^{48}$ These derived differential capacity plots exhibit qualitatively similar features at the potentials found in half-cell experiments for blended NMC $\mid \mathrm{MnO}_{2}$ cathodes and graphite anodes in the literature..$^{25,41}$

With this single degree-of-freedom MSMR model fit to the experimentally-measured usable capacity (over the experimentally-prescribed voltage window), and estimated total capacities of each electrode, it now possible to examine experimentally-relevant half-cell and whole-cell responses over the entire range of capacities (state-of-charge) and cell voltages. Figure 2(a) shows the whole-cell voltage and half-cell potentials of each electrode as a function of incremental charging $(\delta Q)$, where the usable state of charge is simply SOC $(\%)=\frac{\delta Q}{1.48 A h} * 100 \%$ for our fresh cell. At any given cell incremental charge, the whole-cell voltage is the difference between the positive and negative electrode half-cell potentials at the given state of charge. Similarly, Figure 2(b) shows the differential voltage and differential half-cell potentials as a function of cell voltage. In this case, the whole-cell differential voltage is the sum of the half-cell potentials at each given cell potential.

Through our single parameter fit, we are assured that the two end-points of the whole cell charging curve, Figure 2(a), match experiments. However, none of the intermediate points, nor any of the differential voltages, have been shaped by our experiments. Instead, they represent Verbrugge's half-cell data scaled to our cell. Nonetheless, the whole-cell responses in Figs. 2(a) and (b) have all the qualitative features expected from experimental results, with the sharp peaks at $3.5 \mathrm{~V}$ and $3.85 \mathrm{~V}$ associated with the graphite reactions, and the broader peaks at 3.7 and $4.0 \mathrm{~V}$ corresponding to the phase transitions in NMC and LMO, respectively.

Adding model degrees-of-freedom for parameter estimation from whole-cell experiments. - The single degree-of-freedom whole-cell model presented in Figs. 2(a) and (b) is compared to the experimental dataset for a fresh Samsung cell in Figs. 3(a) and 3(b). One sees that the data and model match perfectly at the end points of Fig. 3(a), as enforced by our single model parameter-fit that satisfies Equations (8), (12)(14). Across the whole dataset, the mean absolute error (MAE) between the data and model in Fig. 3(a) is $27 \mathrm{mV}$. Generally, one would like to see a sub-10 mV error between model and fit. Thus, fixing all of Verbrugge et al's parameters (for a NMC 622 composition) does not meet a satisfactory level of fit for this blended NMC 532/LMO cell. 
(a)
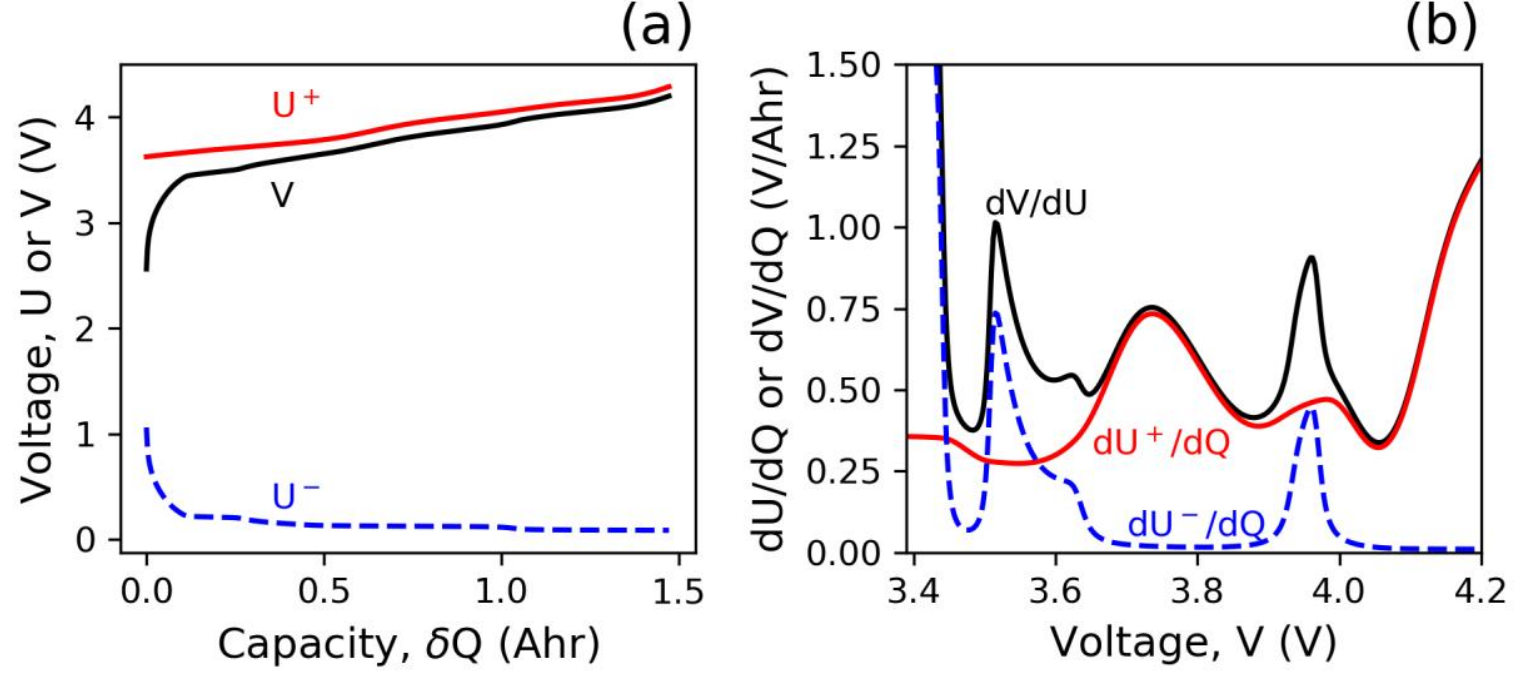

Figure 2: Calculated open circuit potentials and cell voltage (a) and differential potentials and voltage (b) from the MSMR model using thermophysical parameters from the literature, scaled to our cells under test. Whole-cell responses (black) are the difference and sum of the positive (red) and negative (blue) electrodes for the open-circuit and differential voltage, respectively. For the data shown, whole-cell parameters of electrode capacities, N/P ratios, and lithiation windows were estimated to satisfy Equation (12)(14).

The differences between experimental and modeled differential voltages are more dramatic, as seen in Fig. 3(b). While many of the differential voltage peaks are present in both experimental and modeled curves, their locations and relative sizes are quite different. Overall, the MAE for differential voltage is $0.1497 \mathrm{~V} / \mathrm{Ah}$ between the data and model; the results are clearly an inadequate representation of the experimental cell. One of the attributes of the differential forms of the MSMR model (Eq. (2) or its inverse) is that it describes a series of independent insertion reaction "peaks", as in Figure 1, akin to an optical spectrum. In particular, Eq. (2) shows that each of the $j$ insertion reactions has an energy centered at $U_{j}^{0}$, width controlled by $\omega_{j}$, and integrated size set by $Q_{j, t o t}$. Using either a differential capacity or differential voltage formulation, with potential as independent variable, it is fairly intuitive to relax our use of Verbrugge's thermophysical parameters and improve the MAE between experimental and modeled differential voltages. Figures 3(c) and (d) show the consequences of manually manipulating the MSMR parameters to better align the same set of MSMR insertion reaction peaks to the data in Fig. 3(d), without changing any of whole-cell design parameters $\left(\Delta \mathrm{Q}, \mathrm{Q}_{\mathrm{min}}^{ \pm}, \mathrm{Q}_{\mathrm{tot}}^{ \pm}\right)$. 
(a)
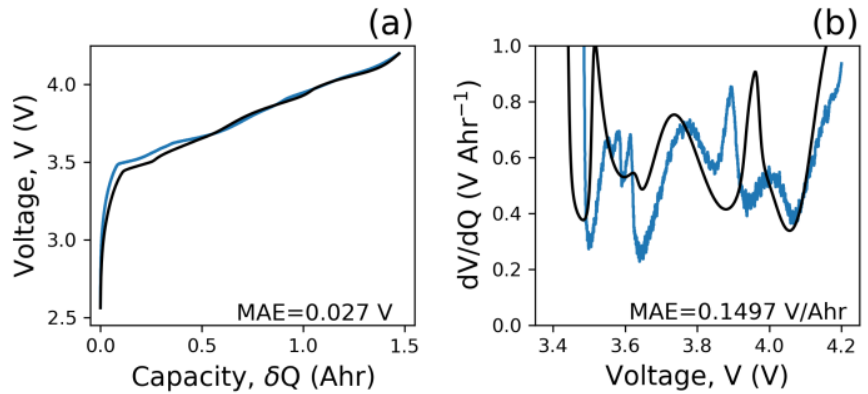

(c)
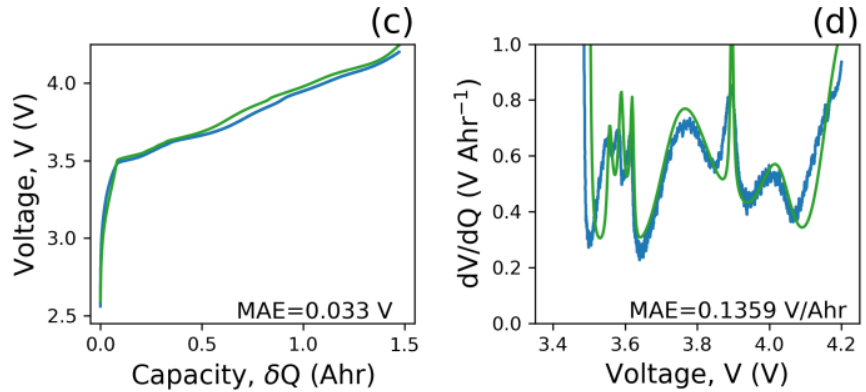

(e)
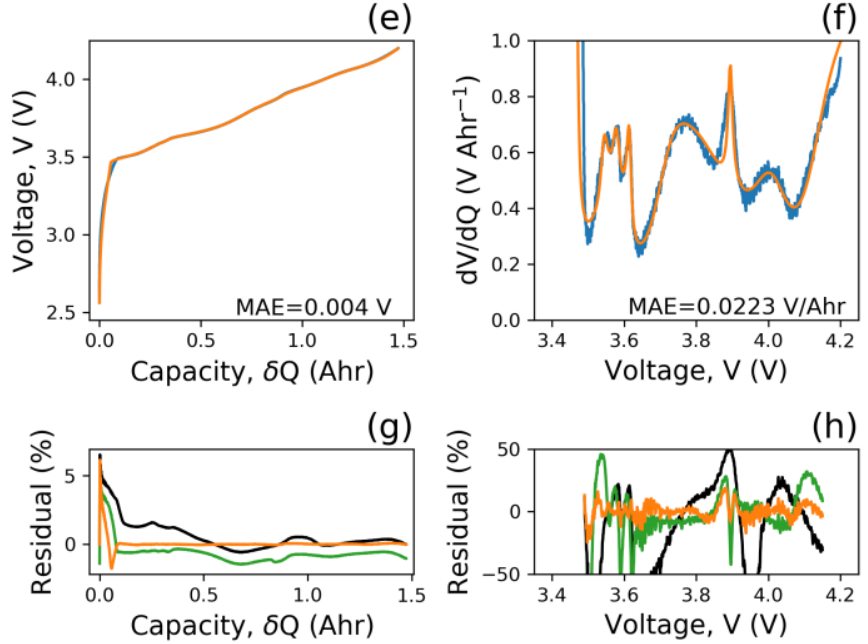

Figure 3 Open circuit potential and differential voltage curves of comparing computed to experimental (blue) data, using initial reaction parameters from literature (a, b) (black), manually modified reaction parameters (c, d) (green), and optimized reaction parameters (e, f) (orange), with their reported mean absolute values. The computed residuals $(\mathrm{g}, \mathrm{h})$ are being compared to the same experimental data collected from a fresh cell that was charged at a $\mathrm{C} / 20$ rate.

A distinct set of modeled features are seen to emerge between Figs. 3(b) and 3(d) through intuitive manipulations of parameters. In particular, three peaks associated with different graphite staging events can be coaxed from the model over a cell voltage range of $3.5 \mathrm{~V}$ to $3.6 \mathrm{~V}$, better matching what is observed experimentally. In the original fits from Verbrugge et al., they noted an inability to capture two small peaks of interest in their experimental data. The parameters involved in the peak emergence included shifting the standard voltages lower, reducing the capacity of these insertion reactions, and decreasing the $\omega_{\mathrm{j}}$ to sharpen 
the peaks of the first four graphite lithiation reactions. A comparison of Figs. 3(b) and (d) also shows that the graphite peak at roughly $4.0 \mathrm{~V}$ has been shifted to a lower voltage to better match experiments by adjusting the capacities $\left(\mathrm{Q}_{\mathrm{j}, \text { tot }}\right)$, so that the phase transition between the last graphite staging reactions occurred earlier in graphite lithiation. Minor adjustments to the standard potentials in the cathode were also made so that the peaks between the model and the experimental data matched better. Qualitatively, these adjusted parameters bring the model much closer into alignment with experimental data, though the quantitative MAE only improves a bit. A consequence of adjusting the reaction parameters and leaving whole-cell design parameters constant is that the voltage limit constraints $\left(V_{\text {upper }}^{\text {exp }}\right.$ and $\left.V_{\text {lower }}^{\text {exp }}\right)$ are no longer met for the 1.48 Ah charge, as seen in Fig. 3(c).. Moreover, the MAE in Fig. 3(c) increased slightly to 33 $\mathrm{mV}$ compared to Fig. 3(a), despite the improved peak shapes in Fig. 3(d).

In short, while the MSMR model is quite intuitive, it is also very challenging to achieve quantitative whole-cell agreement between experimental data and the model for a full charge curve (or discharge curve, see SI) and associated differential voltage curves, while simultaneously meeting the whole-cell constraints represented by Eqs. (8), (12)-(14). Nonetheless, if we hold the number of insertion reactions per electrode fixed at 6 for the anode and 6 for the cathode, per Verbrugge et al., but allow the thermophysical parameters to be degrees of freedom (with bounds), while also letting $\mathrm{Q}_{\min }^{ \pm}$and $\mathrm{Q}_{\text {tot }}^{ \pm}$to be emergent properties that must satisfy Eqs. (8), (12)-(14), then we can find low MAE optimal fits and a set of realistic thermophysical parameters for this specific cell, see Figs. 3(e) and 3(f) and its corresponding parameters in Table 3.

Obtaining best-fit parameters starts with the half-cell modeling data given by Verbrugge, et al., (Figs 3(a) and (b)), with small adjustments to get key features of the differential voltage curves (Fig. 3(c) and (d)). The adjusted half-cell parameters are used as initial guess for fitting the data in Python with the fmin_slsqp (sequential least-squares programming) package in SciPy. The optimization included fitting the differential voltage data between $\mathrm{V}=3.49$ and $\mathrm{V}=4.15 \mathrm{~V}$, with the parameter bounds and voltage limit constraints described in the Methods section. Figures 3(e) and 3(f) show the whole-cell fully optimized fit to data, with equal weighting in Eq. (11). The calculated residual plots for each of the three different parameter sets (Verbrugge et al., tweaked Verbrugge et al., and fully optimized) are displayed as percent difference from the experimental value in Figs. 3 (f) and (g). The residuals are calculated as the deviation between the model and experiment, with the qualitative trends discussed in Figures 3(a)-(f) presented quantitatively through the residuals. One can see the high degree-of-freedom fitting of the MSMR model does an excellent job producing low MAE values in both the voltage and differential analysis results.

Taking the fit parameters and back-calculating the individual half-cell behavior (akin to Fig. 2) produces results comparable to half-cell experiments in the literature for these materials. Estimating half- 
cell potentials from a two-electrode cell, with no reference, can only be known up to an arbitrary constant. However, because the $U_{j}^{0}$ parameters we seed our initial estimation with are derived from referenced halfcell measurements by Verbrugge, et al., and are bounded in the fitting process, the best-fit standard potential estimates are anchored to referenced half-cell potentials. Moreover, with six independent reactions in each half-cell, each constrained to within a neighborhood of $25 \mathrm{mV}$ or less of the initial parameter, we expect our systematic uncertainty in any given $U_{j}^{0}$ values to be comparable to, or smaller than, the scale of our potential bounds. All parameters for the data in Figure 3 are presented in Tables 1 - 3.

Fitting MSMR model parameters to data for degraded cells - As cells cycle, they degrade, as typically manifested by a declining useable capacity between the fixed upper and lower cell voltage limits. The whole-cell modeling paradigm described above allows one to see the evolution of individual parameters of the model, as well as whole-cell emergent phenomenon associated with satisfying overall charge and voltage constraints, i.e., satisfying Eq. (12)-(14) as the cell changes. Figure 4 shows C/20 charging data and optimized MSMR model fits for a fresh cell, Fig 4(a) and (b), a cell cycled 300 cycles at 2C (followed by the $\mathrm{C} / 20$ charge that gets fit, per Methods), Fig. 4(c) and (d), and a cell cycled 600 times, Fig. 4(e) and (f). The 300 cycle cell has a loss of 0.07 Ah of useable capacity (a $4.7 \%$ decline), whereas the 600 cycle cell lost 0.12 Ah of useable (an $8.0 \%$ decline) compared to the fresh cell over the same voltage window.

The model best-fit for each of the state-of-health condition is shown in Fig 4, and the resulting thermophysical parameters are presented in Tables 3-5. Our fits achieve low MAE between the model and each experimental dataset; replicate cell measurements and fits for each state-of-health can be found in the Supporting Information. Initial guesses for fitting aged datasets are the parameters of the previous (less degraded) fit. Just like the fresh-cell scenario, the optimization for the aged fits are done between the voltage range of $3.49 \mathrm{~V}$ and $4.15 \mathrm{~V}$ with equal weighting between the voltage and differential voltage curves.

In all our best-fits, we allow the total capacity of each electrode to be an emergent property of the 6 individual insertion reactions from the electrode, rather than constraining it to a fixed value, like we did in Figs. 1 and 2 (to reduce degrees of freedom). We find that the positive electrode loses capacity as it is cycled, unlike the negative electrode which remains mostly constant, as shown in Table 6.

As the battery degrades, we experimentally observe that the three, distinct graphite peaks at lower voltages begin to lose definition, and eventually coalesce into a single broad peak; this effect is also captured by the MSMR model parameters. We find that the capacity associated with these three early graphite staging insertion reactions is under $15 \%$ of the total electrode capacity, consistent with the literature. ${ }^{27}$. As the battery degrades, we see that the graphite peaks are shifting towards higher potentials, with the most 
(a)
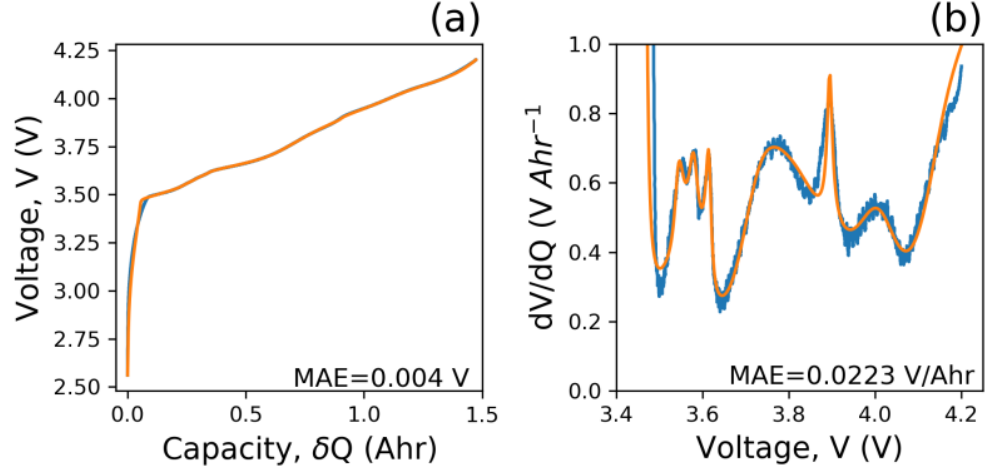

(c)
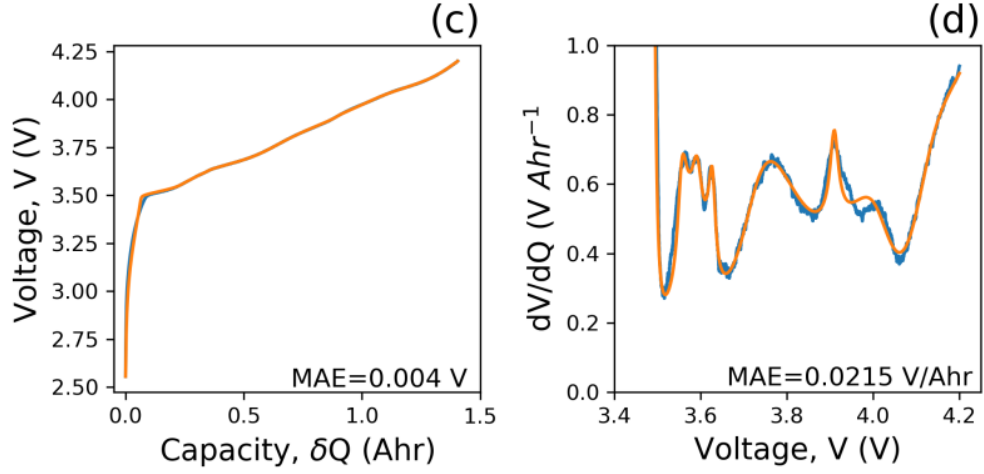

(e)
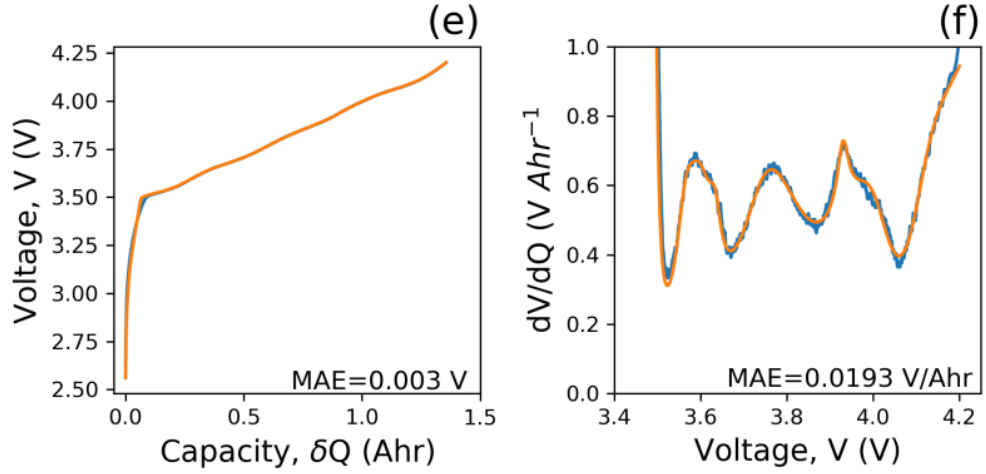

Figure 4 Open circuit potential and differential voltage curves of comparing optimized computed (orange) to experimental (blue) data for fresh cells (a, b), cells that have been aged over 300 cycles (c, d), and cells that have been aged over 600 cycles (e, f), with their reported mean absolute values.

prominent peak at $3.88 \mathrm{~V}$ merging with the LMO transition peak at $4.0 \mathrm{~V}$, potentially indicative of the positive electrode slipping towards higher potentials. The analysis also shows that a large portion of lithium loss in the positive electrode comes from the voltage range between $3.7 \mathrm{~V}$ and $3.8 \mathrm{~V}$. This region involves the transition into a specific rock salt phase, where electrodes with higher nickel content can more easily undergo cation disordering, where lithium and nickel ions can mix, leading to lithium being trapped in the lattice and a nickel ion permanently occupying a lithiation site. ${ }^{25,49}$ 
Capacity loss can be associated with changes in the solid state insertion electrodes $\mathrm{s}^{34,49,52,51,52}$ and/or faradaic inefficiencies. ${ }^{32}$ on one electrode or the other. Most forms of degradation lead to slippage in the electrodes, meaning that the utilization window over which the electrodes are cycling shifts as the battery is being degraded. In differential voltage data, these phenomena can lead to an changes in peak heights in the differential voltage and peak location shifts along the voltage axis associated with voltage slippage. ${ }^{32,53}$

Sensitivity of emergent whole-cell properties on model fitting and experimental noise. - State-ofart commercial batteries often have user or testing agreements that forbid opening the cell to measure parameters such as active material loading, material composition and structure, and specific electrochemical parameters. As Figs. 3 and 4 show, the whole-cell modeling approach described here appears quite useful for disaggregating half-cell thermodynamic behavior from whole-cell experiments, at least when a suitable reference thermophysical dataset is available. The features of the fit that we label "emergent" are the properties that arise from the need to achieve whole-cell charge and voltage constraints, i.e., the variables in Eqs. (8), (12)-(14). Likewise, we include the calculated total capacity for each electrode, Eq. (5), when it emerges from the fit rather than being known from independent experiments. Emergent whole-cell properties are important in cell design, especially for understanding the nature of degradation and its impact on safety, for example, through slippage.

We expect emergent whole-cell properties to be especially sensitive to the robustness of the model optimization process to experimental variation such as noise and data sampling strategy. To test this, we performed a bootstrap analysis on the whole-cell datasets by randomly selecting 1000 points from among the roughly 6000 points in each data set (Figs. 3 and 4 were generated from evenly spaced 1000 points), then replacing and repeating again 500 times. This bootstrap method generates a distribution for every parameter, at every state-of-health tested. The histograms for all these plots can be found in the Supplementary Information. Generally speaking, the bootstrapping results show that the fitting process is quite robust to the noise and spacing of points in our experimental data; the MSMR model converges nicely when the initial physical parameter set is reasonable for the general class of materials being used in the cell. Very few outlier fits were generated.

Figures 5-7 show the bootstrapped histograms for the emergent whole-cell design parameters, such as the maximum capacities for each electrode, $Q_{t o t}^{ \pm}$and the estimated half-cell potentials at the lithiation extremes in each electrode $\left(Q_{\min }^{ \pm}\right.$and $\left.Q_{\max }^{ \pm}\right)$. For these histograms, we show the distribution of each parameter for 0,300 , and 600 cycles, with black dashed lines demarcating the $90 \%$ confidence interval $\left(5^{\text {th }}\right.$ and $95^{\text {th }}$ percentile), and a solid black line denoting the median, or the $50^{\text {th }}$ percentile. 
One insight from performing these bootstraps is to compare their results with capacities from the single fit of evenly spaced points (Table 6). The single fits suggests that the positive electrode's capacity remains largely constant between 0 and 300 cycles, but there is inherently some uncertainty in our fits that is not captured until we employ more statistically driven methods. Figure 5 shows the histogram of the two electrode capacities, $Q_{t o t}^{+}$and $Q_{t o t}^{-}$, calculated from the summation of their respective $Q_{j, t o t}^{+}$for each bootstrap iteration, where we see a systematic shift towards lower positive electrode capacities with aging, whereas the negative electrode narrows into a tightly defined capacity with no significant loss. This behavior is congruent with literature that the positive electrode materials deteriorate at a quicker rate than graphite electrodes, especially more Ni-rich NMCs. ${ }^{51,54,55}$ Moreover, when looking at Figure 1, it is clear that the high density of states provided by staging in the anode means that the sensitivity of the negative electrode half-cell potential is fixed, so achieving the high voltage constraint equations is largely accomplished by slippage in the positive electrode minimum intercalation state.

(a)
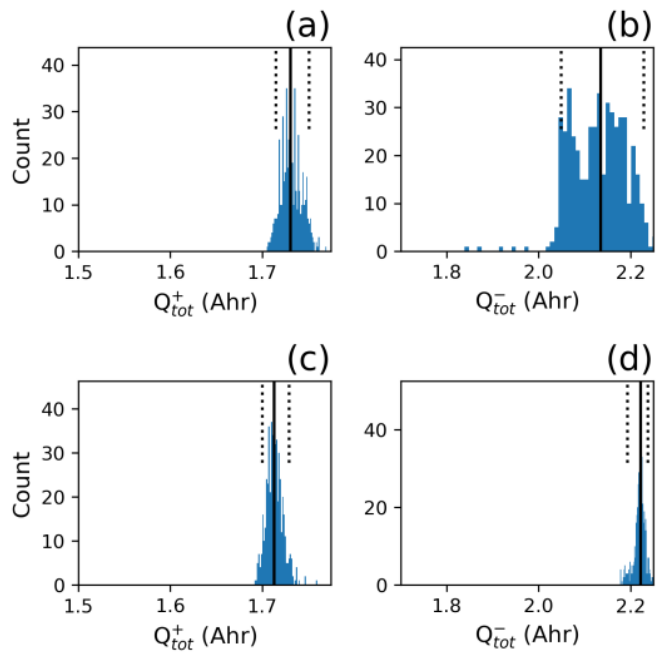

(e)
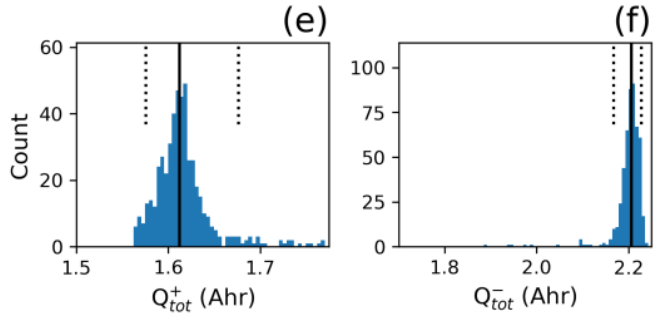

Figure 5 Histograms of each $Q_{\text {tot }}^{+}($left $)$and $Q_{\text {tot }}^{-}($right $)$over the 500 bootstrapped iterations for $0(a, b), 300(c, d)$, and $600(e, f)$ cycles datasets. We show the 5th and 95th percentiles of the values demarcated by the dashed black lines and the median by the solid black line. Histograms showing the same parameter are all plotted along the same $x$-axis for ease for comparison. 
Furthermore, to quantify slippage of the materials, we took each set of fit parameters from the bootstrapped iterations and calculated the potentials at the respective $Q_{\min }^{ \pm}$and $Q_{\text {max }}^{ \pm}$that satisfied $V_{\text {upper }}$ and $V_{\text {lower. }}$ Figure 6 shows the distributions of $Q_{\min }^{+}$and $Q_{\max }^{-}$that satisfy the constraint at top of charge. Here, we do not detect any appreciable differences in the distributions, and this could be expected. Based on our parameters for the graphite electrode (Table 3-5), any usable capacity greater than 1.0 Ahr will have the negative electrode in the final reaction, which can store more than half of the total negative electrode capacity over the half-cell potential window of 0.04 to $0.10 \mathrm{~V}$ vs $\mathrm{Li} / \mathrm{Li}^{+}$. Since the negative electrode capacity does not change appreciably as it is cycled, one finds the half-cell potential one the charged negative electrode does not drift, forcing the positive electrode to also have a nearly constant potential, in order to satisfy whole-cell upper voltage limit of $4.2 \mathrm{~V}$. Examining the capacities $Q_{\max }^{+}$and $Q_{\min }^{-}$for the discharged cell, as shown in Figure 7, shows that the lower voltage constraint we observe is shifting towards higher potentials as the battery degrades, again, aligning with the literature for this chemistry. ${ }^{32,54}$ At the bottom of discharge, the negative electrode is effectively empty, so even minute changes in the capacity could yield large changes in the potential response, whereas the positive electrode is operating in a where an appreciable amount of capacity must be added or removed to induce a potential shift.

(a)
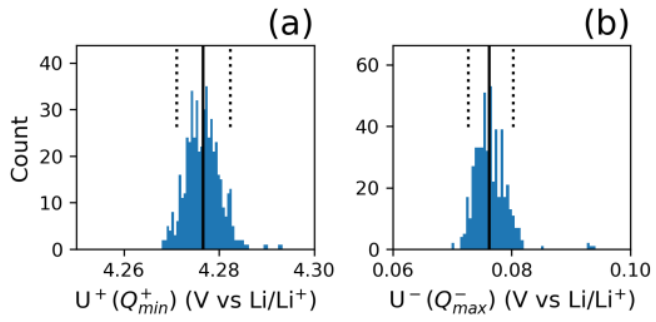

(c)
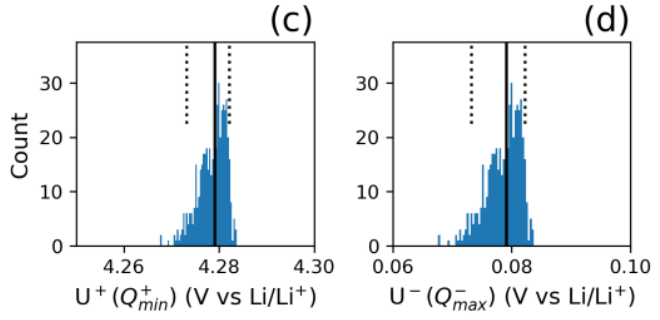

(e)
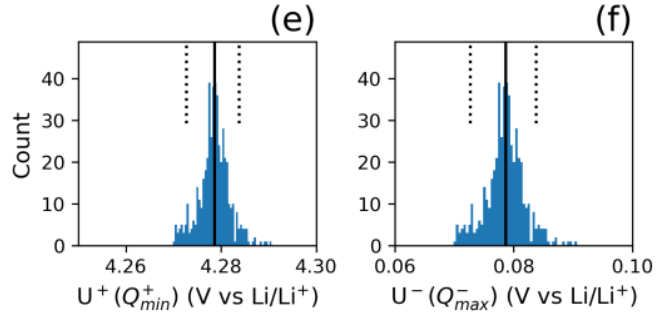

Figure 6 Histograms of each $U^{+}\left(Q_{\min }^{+}\right)$(left) and $U^{-}\left(Q_{\max }^{-}\right)$(right) over the 500 bootstrapped iterations for 0 (a, b), 300 (c, d), and 600 (e, f) cycles datasets, where the difference between the two parameters make up the voltage at the top of charge. We show the 5th and 95th percentiles of the values demarcated by the dashed black lines and the median by the solid black line. Histograms showing the same parameter are all plotted along the same $\mathrm{x}$-axis for ease for comparison. 
(a)
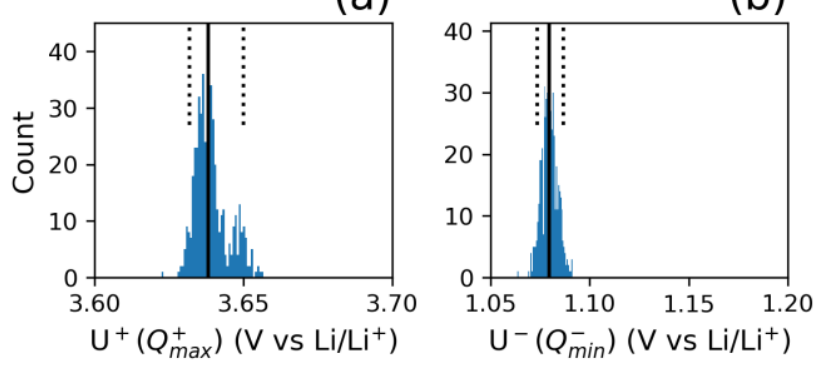

(c)
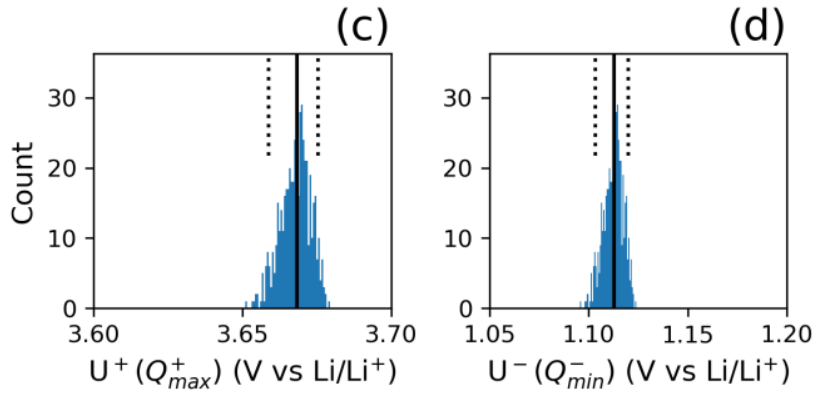

(e)

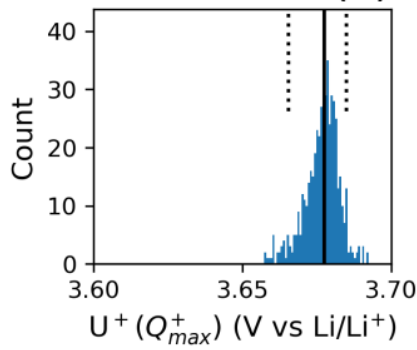

(f)

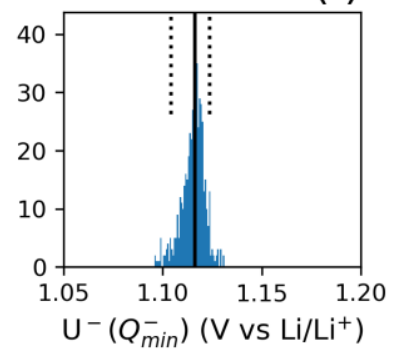

Figure 7 Histograms of each $U^{+}\left(Q_{\max }^{+}\right)$(left) and $U^{-}\left(Q_{\text {min }}^{-}\right)$(right) over the 500 bootstrapped iterations for 0 (a, b), 300 (c, d), and 600 (e, f) cycles datasets, where the difference between the two parameters make up the voltage at the bottom of discharge. We show the 5th and 95th percentiles of the values demarcated by the dashed black lines and the median by the solid black line. Histograms showing the same parameter are all plotted along the same $\mathrm{x}$-axis for ease for comparison.

\section{Conclusion and Implications}

Battery degradation is intrinsically complex, as there are several physical and chemical phenomena that can occur, resulting in electrode imbalance, electrode deformation and degradation, and other modes of loss of lithium inventory. Using open-circuit voltage data in conjunction with differential voltage data can be powerful tools in noninvasively probing these phenomena in fully assembled commercial batteries. While teardown, postmortem analyses, and reassembly of half cells can provide useful insight into the electrode properties and capacity loadings, it is not always feasible or allowed. Here, we extend the easy, generalizable mathematics laid out by the MSMR model to include cell design parameters and the thermodynamic relationships of the two electrodes to allow for the modeling of whole cells. This gives us 
the unique advantage of interpreting the evolution of fundamental reactions and transitions of each electrode, information that is often only extractable from separate half-cell and crystallography studies.

We show that by employing the thermodynamics framework in the MSMR model, we can fit whole-cell experimental thermodynamic data with low errors, allowing us to extract information about the degradative states of the half-cell electrodes without having to perform postmortem testing. Using the MSMR model, we can identify the specific reactions of the NMC, LMO, and the graphite electrodes that are degrading as the cell ages, and how those losses of capacity and deviations from their original one or two-phase behavior affects the resulting thermodynamic response. It is conceivable to perform crystallography experiments in operando to confirm the compositions of each of these phases in cathode materials, to test the validity of the ability of this model to predict how these phase transitions evolve over aging so that future experiments may not need XRD or time-intensive synchrotron experiments. Furthermore, this work strictly focuses on the thermodynamics of the MSMR model, but the framework has been established by Baker et al., to use these principles to model transport and kinetic phenomena, allowing for incredible, fundamental insight opportunities. ${ }^{29,30}$

This present work is aiming to provide the community with a user-friendly tool that allows researchers to gain insights into state-of-health of their cells without having to perform half-cell studies from postmortem procedures. Instead, researchers can utilize a base knowledge of the battery chemistry and the parameters that Verbrugge et al. have published for a variety of electrodes. ${ }^{39}$ It is important to reiterate though, that in the absence of teardown and half-cell data. the MSMR model yields an inherent uncertainty since neither the total capacities nor the true open-circuit potentials are typically known. Additionally, the MSMR model assumes that all reactions are occurring in a uniform electrode, which does not always hold true for degraded electrodes where there could be inhomogeneous particle sizes, cracks, and deformations, which is why we allow for flexibility in the standard potentials to help account for some of these variations. All the data files, code, and instructions on how to use this tool are available on GitHub and Zenodo. ${ }^{38}$

Acknowledgements.-This work has been partially supported by funding from the Boeing-Sutter Endowment, NSF National Research Training Grant fellowship to V.W.H (DGE-1633216), and the Stateof-Washington funded Joint Center for Deployment and Research of Earth Abundant Materials (JCDREAM). This work was performed in the advanced characterization labs at the Washington Clean Energy Testbeds, an open access instrumentation facility managed by the University of Washington Clean Energy Institute. 
Table 1: Initial Parameters from Verbrugge et al.

\begin{tabular}{|c|c|c|c|}
\hline Reaction & $\mathbf{U}_{\mathbf{j}}^{\mathbf{0}}(\mathbf{V} \mathbf{~ v s ~} \mathbf{L i} / \mathbf{L i})$ & $\left.\mathbf{Q}_{\mathbf{j}, \text { tot }} \mathbf{( A h r}\right)$ & $\boldsymbol{\omega}_{\mathbf{j}}$ \\
\hline NMC1 & 3.623 & 0.185 & 0.967 \\
\hline NMC2 & 3.727 & 0.446 & 1.397 \\
\hline NMC3 & 3.906 & 0.290 & 3.505 \\
\hline NMC4 & 4.230 & 0.454 & 5.528 \\
\hline LMO1 & 4.012 & 0.234 & 1.520 \\
\hline LMO2 & 4.149 & 0.191 & 0.930 \\
\hline GRA1 & 0.088 & 0.858 & 0.086 \\
\hline GRA2 & 0.128 & 0.475 & 0.080 \\
\hline GRA3 & 0.143 & 0.297 & 0.725 \\
\hline GRA4 & 0.170 & 0.108 & 2.533 \\
\hline GRA5 & 0.215 & 0.134 & 0.095 \\
\hline
\end{tabular}

Table 2: Parameters from Manual Manipulation for fitting against fresh-cell data

\begin{tabular}{|c|c|c|c|}
\hline Reaction & $\mathbf{U}_{\mathbf{j}}^{\mathbf{0}}(\mathbf{V} \mathbf{~ v s} \mathbf{~ L i} / \mathbf{L i})$ & $\mathbf{Q}_{\mathbf{j}, \text { tot }}(\mathbf{A h \mathbf { h }})$ & $\boldsymbol{\omega}_{\mathbf{j}}$ \\
\hline NMC1 & 3.683 & 0.185 & 0.967 \\
\hline NMC2 & 3.727 & 0.446 & 1.397 \\
\hline NMC3 & 3.906 & 0.290 & 3.505 \\
\hline NMC4 & 4.261 & 0.454 & 5.528 \\
\hline LMO1 & 4.012 & 0.234 & 1.520 \\
\hline LMO2 & 4.169 & 0.191 & 0.930 \\
\hline GRA1 & 0.075 & 1.131 & 0.086 \\
\hline GRA2 & 0.103 & 0.504 & 0.080 \\
\hline GRA3 & 0.124 & 0.049 & 0.150 \\
\hline GRA4 & 0.146 & 0.054 & 0.175 \\
\hline GRA5 & 0.169 & 0.134 & 0.122 \\
\hline
\end{tabular}


Table 3: Parameters from optimization for fresh cell data

\begin{tabular}{|c|c|c|c|}
\hline Reaction & $\mathbf{U}_{\mathbf{j}}^{\mathbf{0}}(\mathbf{V}$ vs $\mathbf{~ L i} / \mathbf{L i})$ & $\mathbf{Q}_{\mathbf{j}, \text { tot }}(\mathbf{A h \mathbf { h }})$ & $\boldsymbol{\omega}_{\mathbf{j}}$ \\
\hline NMC1 & 3.663 & 0.231 & 0.725 \\
\hline NMC2 & 3.746 & 0.407 & 1.072 \\
\hline NMC3 & 3.888 & 0.306 & 2.656 \\
\hline NMC4 & 4.262 & 0.392 & 4.146 \\
\hline LMO1 & 4.019 & 0.222 & 1.472 \\
\hline LMO2 & 4.153 & 0.181 & 0.118 \\
\hline GRA1 & 0.077 & 1.251 & 0.100 \\
\hline GRA2 & 0.108 & 0.549 & 0.185 \\
\hline GRA3 & 0.132 & 0.061 & 0.219 \\
\hline GRA5 & 0.154 & 0.061 & 0.152 \\
\hline GRA6 & 0.177 & 0.165 & 6.828 \\
\hline
\end{tabular}

Table 4: Parameters from optimization for fitting 300 cycles aged cell data

\begin{tabular}{|c|c|c|c|}
\hline Reaction & $\mathbf{U}_{\mathbf{j}}^{\mathbf{0}}(\mathbf{V} \mathbf{~ v s} \mathbf{~ L i} / \mathbf{L i})$ & $\mathbf{Q}_{\mathbf{j}, \text { tot }}(\mathbf{A h \mathbf { h }})$ & $\boldsymbol{\omega}_{\mathbf{j}}$ \\
\hline NMC1 & 3.683 & 0.289 & 0.544 \\
\hline NMC2 & 3.749 & 0.413 & 1.254 \\
\hline NMC3 & 3.908 & 0.260 & 2.560 \\
\hline NMC4 & 4.242 & 0.429 & 3.896 \\
\hline LMO1 & 4.001 & 0.226 & 1.840 \\
\hline LMO2 & 4.137 & 0.136 & 0.948 \\
\hline GRA1 & 0.071 & 1.269 & 0.135 \\
\hline GRA2 & 0.104 & 0.536 & 0.125 \\
\hline GRA3 & 0.129 & 0.054 & 0.182 \\
\hline GRA4 & 0.151 & 0.066 & 0.273 \\
\hline GRA5 & 0.178 & 0.158 & 0.170 \\
\hline
\end{tabular}


Table 5: Parameters from optimization for fitting 600 cycles aged cell data

\begin{tabular}{|c|c|c|c|}
\hline Reaction & $\mathbf{U}_{\mathbf{j}}^{\mathbf{0}}(\mathbf{V} \mathbf{~ v s} \mathbf{~ L i} / \mathbf{L i})$ & $\mathbf{Q}_{\mathbf{j}, \text { tot }}(\mathbf{A h r})$ & $\boldsymbol{\omega}_{\mathbf{j}}$ \\
\hline NMC1 & 3.692 & 0.217 & 0.408 \\
\hline NMC2 & 3.759 & 0.392 & 1.398 \\
\hline NMC3 & 3.918 & 0.221 & 2.452 \\
\hline NMC4 & 4.232 & 0.426 & 3.858 \\
\hline LMO1 & 3.991 & 0.225 & 1.763 \\
\hline LMO2 & 4.134 & 0.140 & 0.896 \\
\hline GRA1 & 0.068 & 1.266 & 0.168 \\
\hline GRA2 & 0.106 & 0.538 & 0.151 \\
\hline GRA3 & 0.129 & 0.054 & 0.228 \\
\hline GRA5 & 0.149 & 0.081 & 0.342 \\
\hline GRA6 & 0.175 & 0.148 & 0.213 \\
\hline
\end{tabular}

Table 6: Capacity of electrodes from fits

\begin{tabular}{|c|c|c|}
\hline Cycles & $\mathbf{Q}_{\text {tot }}^{+}(\mathbf{A h r})$ & $\mathbf{Q}_{\text {tot }}^{-}(\mathbf{A h r})$ \\
\hline $\mathbf{0}$ & 1.740 & 2.168 \\
\hline $\mathbf{3 0 0}$ & 1.753 & 2.181 \\
\hline $\mathbf{6 0 0}$ & 1.619 & 2.180 \\
\hline
\end{tabular}


References

1. M. Armand and J.-M. Tarascon, Nature, 451, 652-657 (2008).

2. S. Chen, C. Niu, H. Lee, Q. Li, L. Yu, W. Xu, J. Zhang, E. J. Dufek, S. M. Whittingham, J. Xiao, J. Lun, Joule, 3, 1094-1105 (2019).

3. B. R. Long, S. G. Rinaldo, K. G. Gallagher, D. W. Dees, S. E. Trask, B. J. Polzin, A. N. Jansen, D. P. Abraham, I. Bloom, J. Bareño, J. R. Croy, J. Electrochem. Soc., 163, A2999 (2016).

4. V. Murray, D. S. Hall, and J. R. Dahn, J. Electrochem. Soc., 166, A329-A333 (2019).

5. M. A. Cabañero, N. Boaretto, M. Röder, J. Müller, J. Kallo, A. Latz., J. Electrochem. Soc., 165, A847 (2018).

6. M. D. Murbach, V. W. Hu, and D. T. Schwartz, J. Electrochem. Soc., 165, A2758-A2765 (2018).

7. I. Bloom, A. N. Jansen, D. P. Abraham, J. Knuth, S. A. Jones, V. S. Battaglia, G. L Henriksen, Journal of Power Sources, 139, 295-303 (2005).

8. C. Liu, Z. G. Neale, and G. Cao, Materials Today, 19, 109-123 (2016).

9. G. Bridgewater, M. J Capener, J. Brandon, M. J. Lain, M. Copley, E. Kendrick, Batteries, 7, 38 (2021).

10. D. P. Abraham, J. L. Knuth, D. W. Dees, I. Bloom, and J. P. Christophersen, Journal of Power Sources, 170, 465-475 (2007).

11. G. Inzelt, ChemTexts, 1, 2 (2014).

12. C. Truchot, M. Dubarry, and B. Y. Liaw, Applied Energy, 119, 218-227 (2014).

13. A. Barai, K. Uddin, M. Dubarry, L. Somerville, A. McGordon, P. Jennings, I. Bloom, Progress in Energy and Combustion Science, 72, 1-31 (2019).

14. C. R. Birkl, E. McTurk, S. Zekoll, F. H. Richter, M. R. Roberts, P. G. Bruce, D. A. Howey, J. Electrochem. Soc., 164, A2644 (2017).

15. C. R. Birkl, E. McTurk, M. R. Roberts, P. G. Bruce, and D. A. Howey, J. Electrochem. Soc., 162, A2271-A2280 (2015).

16. Y. Zhu, T. Gao, X. Fan, F. Han, and C. Wang, Acc. Chem. Res., 50, 1022-1031 (2017).

17. M. Mayur, S. C. DeCaluwe, B. L. Kee, and W. G. Bessler, Electrochimica Acta, 323, 134797 (2019).

18. I. Bloom, L. K. Walker, J. K. Basco, D. P. Abraham, J. P. Christopherson, C. D. Ho, Journal of Power Sources, 195, 877-882 (2010). 
19. J. R. Dahn, Phys. Rev. B, 44, 9170-9177 (1991).

20. T. Motohashi, T. Ono, Y. Sugimoto, Y. Masabuchi, S. Kikkawa, R. Kanno, M. Karppinen, H. Yamauchi, Phys. Rev. B, 80, 165114 (2009).

21. M. S. Whittingham, Chem. Rev., 104, 4271-4302 (2004).

22. J. M. Tarascon, E. Wang, F. K. Shokoohi, W. R. McKinnon, and S. Colson, J. Electrochem. Soc., 138, 2859-2864 (1991).

23. S. Yang, Y. Song, K. Ngala, P. Y. Zavalij, and M. Stanley Whittingham, Journal of Power Sources, 119-121, 239-246 (2003).

24. J. B. Goodenough and Y. Kim, Chem. Mater., 22, 587-603 (2010).

25. M. Dubarry, C. Truchot, M. Cugnet, B. Y. Liaw, K. Gerin, S. Sazhin, D. Jamison, C. Michelbacher, Journal of Power Sources, 196, 10328-10335 (2011).

26. P. Keil and A. Jossen, J. Electrochem. Soc., 164, A6066-A6074 (2017).

27. M. Lewerenz, A. Marongiu, A. Warnecke, and D. U. Sauer, Journal of Power Sources, 368, 57-67 (2017).

28. J. Sieg, M. Storch, J. Fath, A. Nuhic, J. Bandlow, B. Spier, D. U Sauer, Journal of Energy Storage, 30, 101582 (2020).

29. D. R. Baker and M. W. Verbrugge, J. Electrochem. Soc., 165, A3952-A3964 (2018).

30. D. R. Baker, M. W. Verbrugge, and W. Gu, J. Electrochem. Soc., 166, A521 (2019).

31. M. Verbrugge, D. Baker, and X. Xiao, J. Electrochem. Soc., 163, A262-A271 (2016).

32. H. M. Dahn, A. J. Smith, J. C. Burns, D. A. Stevens, and J. R. Dahn, J. Electrochem. Soc., 159, A1405 (2012).

33. S. Lee, J. B. Siegel, A. G. Stefanopoulou, J.-W. Lee, and T.-K. Lee, J. Electrochem. Soc., 167, $090531(2020)$.

34. K. Ando, T. Matsuda, and D. Imamura, Journal of Power Sources, 390, 278-285 (2018).

35. M. W. Verbrugge and B. J. Koch, J. Electrochem. Soc., 150, A374 (2003).

36. D. R. Baker and M. W. Verbrugge, J. Electrochem. Soc., 159, A1341 (2012).

37. J. Kasnatscheew, U. Rodehorst, B. Streipert, S. Wiemers-Meyer, R. Jakelski, R. Wagner, I. C. Laskovic, M. Winter, J. Electrochem. Soc., 163, A2943-A2950 (2016).

38. V. W. Hu and D. T. Schwartz, (2021) https://zenodo.org/record/5542603. 
39. M. Verbrugge, D. Baker, B. Koch, X. Xiao, and W. Gu, J. Electrochem. Soc., 164, E3243E3253 (2017).

40. X. Feng, Y. Merla, C. Weng, M. Ouyang, X. He, B. Y. Liaw, S. Santhanagopalan, X. Li, P. Liu, L. Lu, X. Han, D. Ren, Y. Wang, R. Li, C. Jin, P. Huang, M. Yi, L. Wang, Y. Zhao, Y. Patel, G. Offer, eTransportation, 3, 100051 (2020).

41. Z. Mao, M. Farkhondeh, M. Pritzker, M. Fowler, and Z. Chen, J. Electrochem. Soc., 164, A3469-A3483 (2017).

42. N. Nitta, F. Wu, J. T. Lee, and G. Yushin, Materials Today, 18, 252-264 (2015).

43. R. Weber, C. R. Fell, J. R. Dahn, and S. Hy, J. Electrochem. Soc., 164, A2992 (2017).

44. J. Zheng, P. Yan, J. Zhang, M. H. Engelhard, Z. Zhu, B. J. Polzin, S. Trak, J. Xiao, C. Wang, J. Zhang, Nano Research, 10 (2017).

45. S.-K. Jung, H. Gwon, J. Hong, K. Park, D. Seo, H. Kim, J. Hyun, W. Yang, K. Kang, Advanced Energy Materials, 4, 1300787 (2014).

46. Y. Zhang, H. Xie, H. Jin, Q. Zhang, Y. Li, X. Li, K. Li, C. Bao, IOP Conf. Ser.: Earth Environ. Sci., 603, 012051 (2020).

47. T. Ohzuku, M. Kitagawa, and T. Hirai, J. Electrochem. Soc., 137, 769-775 (1990).

48. K. G. Gallagher, D. W. Dees, A. N. Jansen, D. P. Abraham, and S.-H. Kang, J. Electrochem. Soc., 159, A2029-A2037 (2012).

49. T. Li, X. Yuan, L. Zhang, D. Song, K. Shi, C. Bock, Electrochem. Energ. Rev., 3, 43-80 (2020).

50. A. Tornheim and D. C. O’Hanlon, J. Electrochem. Soc., 167, 110520 (2020).

51. J. P. Pender, G. Jha, D. H. Youn, J. M Ziegler, I. Andoni, E. J Choi, A. Heller, B. S. Dunn, P. S. Weiss, R. M. Penner, C. B. Mullins, ACS Nano, 14, 1243-1295 (2020).

52. D. H. Jang, Y. J. Shin, and S. M. Oh, J. Electrochem. Soc., 143, 2204-2211 (1996).

53. M. Dubarry, C. Truchot, and B. Y. Liaw, Journal of Power Sources, 219, 204-216 (2012).

54. J. A. Gilbert, J. Bareño, T. Spila, S. E. Trask, D. J. Miller, B. J Polzin, A. N. Jansen, D. P. Abraham, J. Electrochem. Soc., 164, A6054-A6065 (2017).

55. D. Zhang, B. S. Haran, A. Durairajan, R. E. White, Y. Podrazhansky, B. N. Popov, Journal of Power Sources, 91, 122-129 (2000). 
